\title{
Absence of QTc Prolongation with Sodium N-(8-[2- Hydroxybenzoyl] Amino) Caprylate (SNAC), an Absorption Enhancer Co-Formulated with the GLP- 1 Analogue Semaglutide for Oral Administration
}

\author{
Charlotte Granhall • Tine A. Bækdal • Astrid Breitschaft • \\ Flemming L. Søndergaard · Thomas W. Anderson · Mette Thomsen
}

Received: February 19, 2021 / Accepted: June 23, 2021 / Published online: July 28, 2021

(C) The Author(s) 2021

\begin{abstract}
Introduction: Oral delivery of proteins, including glucagon-like peptide 1 (GLP-1) receptor agonists, is impeded by low gastrointestinal permeation. Oral semaglutide has been developed for once-daily oral administration by co-formulation of the GLP-1 analogue semaglutide with an absorption enhancer, sodium $\mathrm{N}$-(8-[2-hydroxybenzoyl] amino) caprylate (SNAC, $300 \mathrm{mg}$ ). A randomised, partially double-blind, placebo-controlled thorough QT/corrected QT (QTc) trial was conducted to confirm the absence of unacceptable QTC interval prolongation with SNAC. QT is defined as interval on the electrocardiogram, measured from the start of the QRS complex to the end of the T wave.

Methods: Part A of the study sought to identify an appropriate dose of SNAC (which was substantially higher than that used in the oral semaglutide co-formulation) for QTc assessment. Three sequential healthy volunteer cohorts were randomised to escalating single oral doses of SNAC $(1.2,2.4$ or $3.6 \mathrm{~g})$ or placebo. Following identification of an appropriate dose,
\end{abstract}

C. Granhall · T. A. Bækdal (凹) · F. L. Søndergaard .

T. W. Anderson $\cdot$ M. Thomsen

Novo Nordisk A/S, Søborg, Denmark

e-mail: tabq@novonordisk.com

A. Breitschaft

Parexel International GmbH, Berlin, Germany a cross-over trial was conducted (Part B). Healthy volunteers received one of four treatment sequences, including single oral doses of SNAC, moxifloxacin (positive control) and placebo. Primary objectives were to (1) assess adverse events (AEs) with escalating SNAC doses and (2) confirm that SNAC does not cause unacceptable QTc interval prolongation versus placebo, using the Fridericia heart rate-corrected QT interval (QTcF).

Results: All subjects completed Part A $(N=36)$ and 46 subjects completed Part B. In Part A, all AEs were mild to moderate in severity; no relationship was identified between $\mathrm{AE}$ incidence and SNAC dose. SNAC $3.6 \mathrm{~g}$, the maximum investigated SNAC dose, was selected for Part B. There was no unacceptable prolongation of the QTcF interval with SNAC $3.6 \mathrm{~g}$, and assay sensitivity was demonstrated with moxifloxacin as the positive control. There was no significant exposure-response relationship between SNAC concentration and QTcF interval, and no instances of QTc interval $>450 \mathrm{~ms}$ or increases $>30 \mathrm{~ms}$.

Conclusion: This QT/QTc trial demonstrates that SNAC doses 12-fold higher than the $300 \mathrm{mg}$ dose used in the oral formulation of semaglutide do not cause unacceptable prolongation of the QTcF interval.

Trial Registration: Clinicaltrials.gov identifier: NCT02911870. 


\section{PLAIN LANGUAGE SUMMARY}

Medications that are taken orally can be broken down by acid in the stomach before they are absorbed and therefore be less effective. Oral semaglutide is a novel type 2 diabetes medication that is formulated with the absorption enhancer sodium N-(8-[2-hydroxybenzoyl] amino) caprylate (SNAC), which helps to protect against semaglutide degradation in the stomach. Regulatory authority guidelines recommend that new therapies should be tested for prolongation of the QT interval, an important part of the heart's electrical cycle. A previous trial demonstrated that semaglutide alone, which is currently available as an injectable diabetes therapy, did not prolong the QT interval when given in doses higher than those used in patients. Therefore, the current trial was conducted to assess whether the SNAC component of oral semaglutide has any relevant prolonging effect on the QT interval. Following regulatory guidelines for trials evaluating prolongation of the QT interval, the first part of the trial aimed to find a suitably high dose of SNAC. The second part of the trial aimed to confirm that SNAC does not prolong the QT interval. The results of this trial demonstrated that a $3.6 \mathrm{~g}$ dose of SNAC, which is 12-fold higher than the amount contained in oral semaglutide, does not prolong the QT interval. The safety and tolerability of SNAC $1.2 \mathrm{~g}, 2.4 \mathrm{~g}$ and $3.6 \mathrm{~g}$ were assessed in this trial and no concerns were identified. These results, taken alongside those of the previous QT interval study with subcutaneous semaglutide, indicate no relevant effect of oral semaglutide on the QT interval.

Keywords: Cardiac repolarisation; Drug safety; QT interval; SNAC

\section{Key Summary Points}

\section{Why carry out this study?}

It is important to understand whether new therapies can impact the duration of cardiac ventricular depolarisation and repolarisation and lead to arrhythmias.

Oral semaglutide contains an absorption enhancer, sodium $\mathrm{N}$-(8-[2-

hydroxybenzoyl] amino) caprylate (SNAC), and the potential cardiac effects of SNAC require evaluation.

The effect of SNAC at concentrations substantially higher than those used in oral semaglutide were assessed in healthy volunteers to determine whether there was an effect on the QTc interval on an electrocardiogram.

\section{What was learned from the study?}

Doses of SNAC substantially higher than that used in oral semaglutide did not result in unacceptable prolongation of the QTc interval.

Along with previous data showing no impact of supratherapeutic doses of subcutaneous semaglutide on QTC interval, these data suggest that oral semaglutide does not cause unacceptable prolongation of the QTc interval.

\section{INTRODUCTION}

Oral delivery of protein-based drugs is hindered by their limited permeation across the gastrointestinal tract and rapid enzymatic and $\mathrm{pH}$ induced degradation in the stomach. Co-formulation of therapeutic peptides with absorption enhancers can be used to facilitate oral delivery.

Glucagon-like peptide 1 (GLP-1) receptor agonists provide an effective treatment option 
for patients with type 2 diabetes [1], with currently available formulations administered by subcutaneous (s.c.) injection [2-7]. An orally available formulation offers the potential of earlier treatment initiation and may improve patient acceptance and adherence. The human GLP-1 analogue semaglutide is now approved as both a subcutaneous injection (as Ozempic) [5] as well as an oral formulation (as Rybelsus) [8] in European countries, Japan and the USA, among others. To allow oral administration, oral semaglutide is co-formulated with the absorption enhancer, sodium $\mathrm{N}$-(8-[2-hydroxybenzoyl] amino) caprylate (SNAC), a small fatty acid derivative that protects semaglutide against enzymatic degradation through a localised increase in $\mathrm{pH}$ and transiently enhances its absorption across the gastric epithelium via the transcellular route [9-11]. During the development of oral semaglutide, SNAC was studied extensively in non-clinical and clinical studies (including the present study), to investigate potential drug-drug interactions, pharmacokinetics and general safety. SNAC has also been formulated with vitamin B12 [12] and used as a medical food to treat vitamin B12 deficiency anemia [13].

The QT or corrected QT (QTc) interval on the surface electrocardiogram (ECG) represents the duration of cardiac ventricular depolarisation and subsequent repolarisation. As QT/QTc interval prolongation, and hence delay of ventricular repolarisation, may be associated with life-threatening arrhythmias, regulatory authority guidelines require that all novel therapeutic agents with systemic exposure are evaluated for their impact on the QT/QTc interval [14]. Where possible, these guidelines recommend testing drugs at levels substantially exceeding the anticipated maximum therapeutic exposure. A previous study showed that s.c. semaglutide at supratherapeutic doses does not result in a QTc interval prolongation in healthy subjects [15]. The level of exposure achieved with s.c. semaglutide is also expected to be supratherapeutic for the oral formulation. However, the effect of SNAC alone on QTC interval has not previously been investigated. This thorough QT/QTc trial was conducted to confirm that there is no unacceptable prolongation of the QTc interval with SNAC. The trial included doses 12-fold higher than the SNAC $300 \mathrm{mg}$ dose used in the oral formulation of semaglutide.

\section{METHODS}

\section{Study Design}

The trial was conducted in accordance with local regulatory requirements, good clinical practice guidelines and the Declaration of Helsinki $[16,17]$. Approval from the Institutional Review Board/Independent Ethics Committee (Landesamt für Gesundheit und Soziales Berlin, Ethik-Kommission des Landes Berlin, Berlin) was obtained prior to the commencement of the trial. All subjects provided written informed consent before any trial-related activity.

This was a two-part trial, encompassing a dose-finding study (Part A) followed by a QTc assessment study (Part B) (NCT02911870). For both parts A and B, subjects were randomised at visit 2 , day 1 . Treatment allocation took place according to a subject randomisation list provided by the trial sponsor, and when a subject was randomised, they were assigned the lowest available randomisation number.

The article was performed in accordance with the ethical standards of the institutional and/or national research committee and with the 1964 Helsinki declaration and its later amendments or comparable ethical standards.

\section{Part A (Dose Finding)}

Part A was a single-centre, single-dose, randomised, partially double-blind (dose level not blinded), placebo-controlled study conducted to assess the safety profile of doses of SNAC (Emisphere Technologies, Roseland, NJ, USA) exceeding that contained in oral semaglutide. A further aim of Part A was to identify the appropriate dose for use in the QTc assessment. A sequential cohort study design was used with three escalating SNAC doses. The decision to proceed with each escalation was based on evaluation of the safety and pharmacokinetics of the prior dose by the trial safety group. After screening, subjects in the first cohort were 


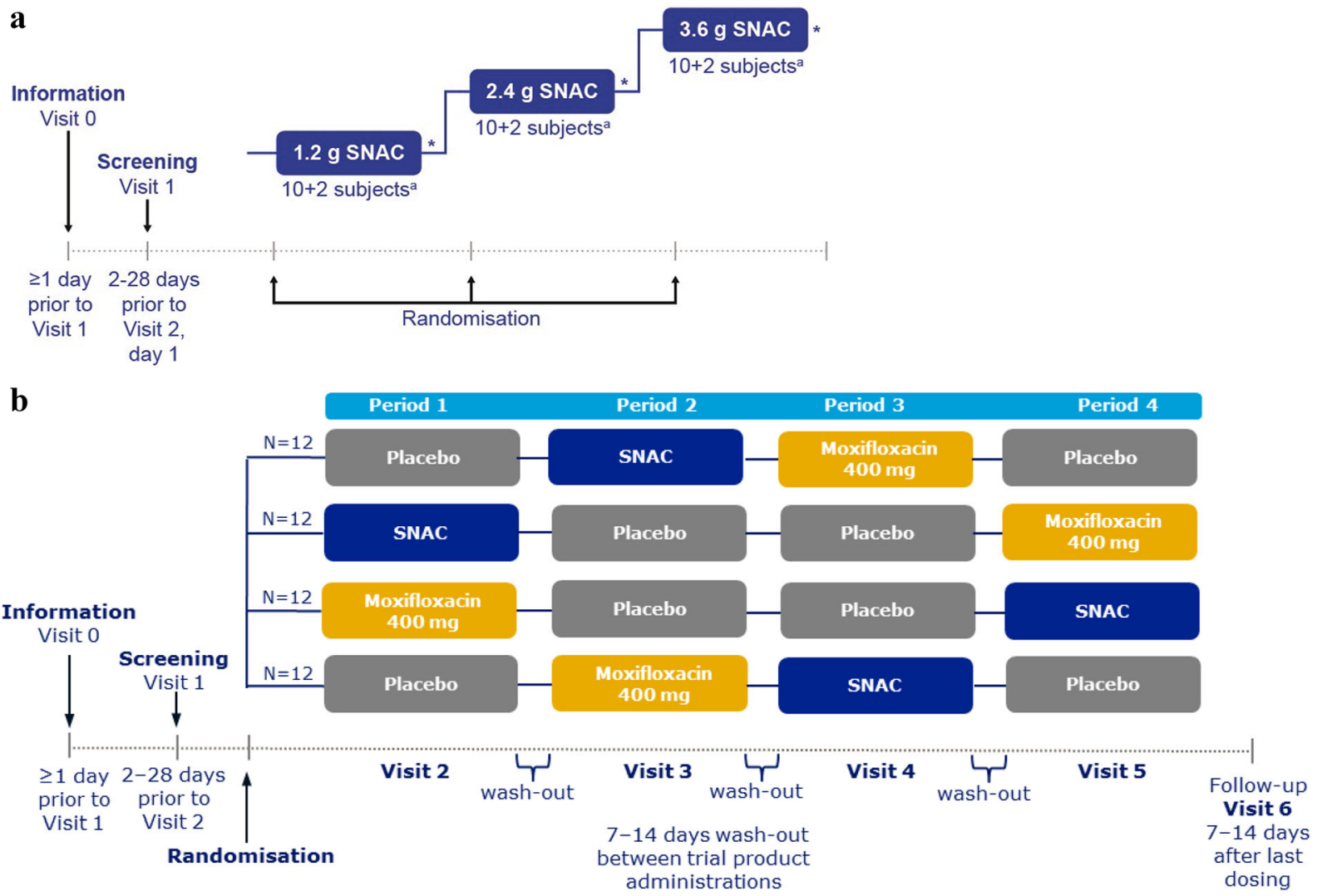

Fig. 1 Trial design for Part A (a) and Part B (b). Asterisk $\left.{ }^{*}\right)$ denotes trial safety group review. ${ }^{a}$ Ten subjects on SNAC and two subjects on placebo at each dose level. For each dose level: randomisation, dosing and

randomised to receive a single oral dose of SNAC $1.2 \mathrm{~g}$ administered as four $300 \mathrm{mg}$ tablets or placebo. Subsequent cohorts of subjects were randomised to receive single oral doses of SNAC $2.4 \mathrm{~g}$ (eight $300 \mathrm{mg}$ tablets) or placebo, and SNAC $3.6 \mathrm{~g}$ (twelve $300 \mathrm{mg}$ tablets) or placebo. Within each sequential cohort, subjects were randomised 5:1, with ten subjects receiving SNAC and two receiving placebo (Fig. 1a).

\section{Part B (QTc Assessment)}

Part B was a single-centre, single-dose, randomised, partially double-blind (moxifloxacin not blinded), placebo- and positive-controlled, cross-over trial conducted to evaluate the effect of a dose of SNAC exceeding that contained in oral semaglutide on QTc interval. To determine the appropriate dose of SNAC for Part B, the trial pharmacokinetics sampling (visit 2). Follow-up (visit 3) occurred 7-10 days after dosing. SNAC Sodium $\mathrm{N}$-(8-[2hydroxybenzoyl] amino) caprylate

safety group reviewed data from Part A and selected the maximum SNAC dose that was not associated with clinically relevant safety findings or tolerability issues deemed likely to influence QT/QTc assessment.

Subjects were randomised to receive one of four different treatment sequences as part of a four-period cross-over Williams design. Each sequence included: a single oral dose of SNAC; a single oral dose of moxifloxacin $400 \mathrm{mg}$ (positive control); and two single doses of placebo. Each period was separated by a washout period of 7-14 days (Fig. 1b), which was considered appropriate given the elimination half-life of moxifloxacin is $11.5-15.6 \mathrm{~h}$ and the elimination half-life of SNAC is approximately $2 \mathrm{~h}$. The cross-over design was chosen as this allows subjects to serve as their own controls, thus reducing the sample size. The inclusion of two 
placebo treatment periods also reduces sample size while maintaining statistical power and fewer subjects are required to take the active drugs compared to a design with one placebo period $[18,19]$. Another benefit is that there are twice as much data available to perform the population-specific (accounted for individual subject levels) corrected QT interval (QTcP) correction, since this method uses (QT, RR)pairs from off-drug data (placebo) from within the trial, making it more robust. RR is defined as the interval in the ECG, measured from the peak of one $\mathrm{R}$ wave to the peak of the next $\mathrm{R}$ wave.

All study drugs were administered in the morning following fasting for $\geq 6 \mathrm{~h}$. Study drugs were swallowed with $240 \mathrm{~mL}$ of water with fasting maintained for $4 \mathrm{~h}$ post-dose and water restricted for $2 \mathrm{~h}$ post-dose. Subjects were not permitted to use prescription or non-prescription medications from screening to $12 \mathrm{~h}$ after the last dose, except for routine vitamins, acetylsalicylic acid and paracetamol.

\section{Study Population}

Eligible participants included healthy male subjects aged $18-55$ years with body mass index (BMI) between 18.5 and $28.0 \mathrm{~kg} / \mathrm{m}^{2}$ and normal ECG. Only male subjects were included in this trial due to the practical challenges associated with fitting ECG electrodes to females. Subjects with sitting blood pressure at screening (after resting for at least $5 \mathrm{~min}$ ) outside the range of 90-139 mmHg for systolic blood pressure or $50-89 \mathrm{mmHg}$ for diastolic blood pressure were excluded. Other exclusion criteria included heart rate (HR) outside the range $45-89$ beats per minute (bpm), smoking, history of major gastric surgery potentially affecting absorption of the trial product and recent blood donations. Subjects randomised in Part A were not eligible for randomisation in Part B.

\section{Study Objectives}

For Part A (dose finding), the primary objective was to assess the safety and tolerability of doses of SNAC exceeding the dose contained in oral semaglutide, in terms of the number of adverse events (AEs). Secondary objectives included investigation of pharmacokinetic properties of SNAC. For Part B (QTc assessment), the primary objective was to confirm that a dose of SNAC exceeding that contained in the oral semaglutide tablet does not cause an unacceptable prolongation of the QTc interval compared with placebo, assessed using Fridericia HR-corrected QT interval (QTcF) at 14 time points (co-primary endpoints) ranging from $10 \mathrm{~min}$ to $12 \mathrm{~h}$ after trial product administration. Supportive secondary endpoints included QTcP, HR, duration of QRS complex (deflections in the ECG that denote depolarisation of the ventricles, between the beginning of the $\mathrm{Q}$ wave and the end of the $S$ wave), PR interval (the interval in the ECG from the start of the P wave to the start of the QRS complex), uncorrected QT interval and occurrence of $\mathrm{T}$ - and U-wave abnormalities, absolute QTc interval prolongations > $450,>480$ or $>500 \mathrm{~ms}$, QTc interval increases from baseline $>30$ or $>60 \mathrm{~ms}$, HR $>100 \mathrm{bpm}$, PR interval $>200 \mathrm{~ms}$, or $\mathrm{QRS}>100 \mathrm{~ms}$. Other supportive secondary endpoints included assessment of the pharmacokinetic properties of SNAC and treatment-emergent AEs.

\section{Study Assessments}

\section{Electrocardiogram Assessments}

On the day of trial product administration, ECGs were recorded continuously using US Food and Drug Administration-approved 12lead Holter recorders. ECG recordings commenced $1 \mathrm{~h}$ prior to dosing and continued until $\geq 12 \mathrm{~h}$ post-dose. Subjects were required to lie supine for $15 \mathrm{~min}$ prior to each planned ECG recording time point to allow the HR to stabilise. The QT interval was measured in a blinded manner at the ECG core laboratory, with semi-automatic extraction of intervals, and all interval determinations for a given subject were performed by a single technician. For the co-primary endpoint analyses in Part B, QT correction was performed using Fridericia's correction, with QTcF calculated as QT $/ \sqrt[3]{\mathrm{RR}}$, where QT and RR are in seconds. For each ECG time point, QTc was calculated based on 
triplicate ECGs, with each including four cardiac cycles. Averages of the four QT intervals and four RR intervals were calculated for each ECG and subsequently used to calculate QTcF. The resulting three QTcF intervals per time point were averaged and the output used for the statistical analysis.

\section{Pharmacokinetic Assessments}

Blood samples for the pharmacokinetic analysis were collected pre-dose and every $10 \mathrm{~min}$ for the first hour post-dose, and then at 1, 1.5, 2, $2.5,3,4,5,8$ and $12 \mathrm{~h}$ post-dose. Pharmacokinetic analyses for SNAC included determination of area under the SNAC plasma concentration-time curve from 0 to $12 \mathrm{~h}$ post-dose $\left(\mathrm{AUC}_{0-12 \mathrm{~h}}\right)$, maximum observed SNAC plasma concentration $\left(C_{\max }\right)$, time to $C_{\max }\left(t_{\max }\right)$ and terminal half-life of SNAC $\left(t_{1 / 2}\right)$. SNAC metabolites were also analysed.

\section{Other Assessments}

Additional assessments included recording of AEs and vital signs, and blood sampling for biochemistry and haematology. In Part A, arterial lactate and arterial blood gas analysis were included.

\section{Statistical Methods}

For the QTc assessment, sample size was calculated to achieve a $90 \%$ power to confirm that all of the 14 time-matched mean treatment differences in baseline-adjusted QTcF between SNAC and placebo were below $10 \mathrm{~ms}$ using a one-sided test with a 5\% significance level. Assuming a standard deviation (SD) of $10 \mathrm{~ms}$ of baselineadjusted QTcF, a within-treatment period correlation of 0.5 and a prolonging effect of SNAC on QTcF at the 14 time points (assumed peak effect of $4 \mathrm{~ms}$ ), 41 subjects who completed the study were required to achieve at least 90\% power. To account for subjects withdrawing and to have an equal number of subjects randomised to each of the four treatment sequences, 48 subjects were randomised.

Each of the 14 QTcF co-primary endpoints was entered as a dependent variable in an analysis of covariance model, with subject, period and treatment as fixed effects and treatment period-specific baseline QTcF as covariate. Time-matched mean baseline-adjusted treatment differences (SNAC minus placebo) were estimated for each of the 14 post-dose time points and presented together with two-sided 90\% confidence intervals (CI) and $p$ values for the one-sided test of a mean difference $\geq 10 \mathrm{~ms}$.

Assay sensitivity was considered confirmed if differences between the positive control (moxifloxacin) and placebo in any of the three QTcF endpoints measured at 2, 3 and $4 \mathrm{~h}$ post-dosing (analysed using an analysis of covariance model similar to that described above) were shown to be above $5 \mathrm{~ms}$ (in accordance with ICH E14 [14]), tested one sided with an overall significance level of $5 \%$ and with Bonferroni correction for multiple testing. The anticipated number of subjects who completed the study would provide approximately $90 \%$ power to establishing assay sensitivity.

QTcP was calculated as QT/RR ${ }^{\beta}$, where $\beta$ is estimated based on all (QT, RR)-pairs from the two placebo treatment periods by use of the parabolic model $\mathrm{QT}=\alpha \mathrm{RR}^{\beta}$. The parabolic model was fitted by log-transforming QT and using it as the dependent variable in a linear normal mixed-effect model with log-transformed RR as a continuous fixed effect and as a random effect, an intercept as fixed effect and as a random effect, and an interaction between subject and period as random effect. The covariance structure of the intercept and slope for log-transformed RR was unstructured. From this model the estimated parameter (slope) for log-transformed RR as continuous fixed effect was used as $\beta$ to calculate QTcP.

Secondary ECG endpoints were analysed in a manner similar to the analysis of the co-primary QTcF endpoints, with the exception that $p$ values were not presented. Two exposure-response (concentration-QTcF) analyses were performed using linear normal mixed-effect models. SNAC AUC $_{0-12 \mathrm{~h}}$ and $C_{\max }$ endpoints were analysed using an analysis of variance model with the log-transformed endpoint as dependent variable and treatment level as the fixed factor. Dose proportionality was assessed using linear regression models, with the coefficient for logtransformed SNAC dose (defined as $\beta$ ) estimated 
and presented as $2^{\beta}$ (the increase in exposure when the dose is doubled) with a corresponding 95\% CI. Each model was used to predict the treatment difference in baseline-adjusted QTcF between SNAC and placebo and corresponding $90 \% \mathrm{CI}$ at the mean $C_{\max }$ of SNAC. Descriptive statistics were used for other pharmacokinetic endpoints and for other continuous endpoints. Analyses of ECG and pharmacokinetic endpoints were based on the full analysis set (all subjects randomised and receiving $\geq 1$ dose of study drug); analyses of safety were performed using the safety analysis set (all subjects receiving $\geq 1$ dose of study drug).

\section{RESULTS}

\section{Participants}

A total of 36 subjects were randomised and completed Part A ( $N=10$ for each of the three SNAC doses; $N=6$ for placebo). Mean (SD) age was 40 (10) years and mean BMI was 24.7 (1.8) $\mathrm{kg} / \mathrm{m}^{2}$. Forty-eight subjects were randomised and 46 completed Part B $(N=12$ per treatment sequence); of the two subjects who did not complete the trial, one withdrew due to an $\mathrm{AE}$ and one was withdrawn by the investigator. All 48 patients were included in the full analysis and safety sets. Mean (SD) age of the participants in Part B was 35 (9) years and mean BMI was $24.7(2.4) \mathrm{kg} / \mathrm{m}^{2}$. All subjects were white except for one mixed-race subject in Part $A$ and one Black/African-American subject in Part B.

\section{Part A-Dose Finding}

Treatment-emergent AEs were reported by two of ten subjects (2 AEs) with SNAC $1.2 \mathrm{~g}$, by one of ten subjects with SNAC $2.4 \mathrm{~g}$ (3 AEs), by two of ten subjects with SNAC $3.6 \mathrm{~g}$ (4 AEs) and by two of six subjects with placebo (4 AEs). All AEs were mild or moderate in severity. The most frequently reported AEs $(N=5)$ were gastrointestinal disorders, including diarrhoea, nausea and vomiting, which occurred in four subjects. The number of AEs reported was low and appeared to be similar across treatment groups. There did not appear to be a relationship between SNAC dose and number of AEs reported. No clinically relevant changes from baseline to end of treatment were observed in HR, arterial blood gas parameters (including lactate) or other safety assessments.

Pharmacokinetic analyses revealed that the $\mathrm{AUC}_{0-12 \mathrm{~h}}$ increased with increasing dose of SNAC, consistent with dose proportionality $\left(2^{\beta}\right.$ : 2.12 [95\% CI 1.80, 2.50]). $C_{\max }$ increased with increasing dose of SNAC, but less than dose proportional $\left(2^{\beta}: 1.46\right.$ [95\% CI 1.07, 2.00]). $t_{\max }$ appeared to increase with increasing dose of SNAC, with median values ranging from approximately $25 \mathrm{~min}$ to $1.5 \mathrm{~h}$. $t_{1 / 2}$ was similar for the three doses of SNAC, with a geometric mean of $1.9 \mathrm{~h}$.

As no safety or tolerability concerns were identified after single dosing with any of the three SNAC doses assessed, the maximum dose (3.6 g) was selected for use in Part B.

\section{Part B-QTc Assessment}

The QTc assessment mean outcome values are shown in Table 1. The treatment difference in baseline-adjusted QTcF interval between moxifloxacin and placebo was $>5 \mathrm{~ms}$ at all predefined time points $(2,3$ and $4 \mathrm{~h} ; p<0.001$ at all three time points $[p$ value for the one-sided test of the null hypothesis $\left(H_{0}\right)$ of a mean difference $\leq 5 \mathrm{~ms}$ against an alternative hypothesis $\left(H_{\mathrm{A}}\right)$ of a mean difference $\left.\left.>5 \mathrm{~ms}\right]\right)$, thereby confirming assay sensitivity.

The treatment difference in the baseline-adjusted QTcF interval between SNAC $3.6 \mathrm{~g}$ and placebo was significantly $<10 \mathrm{~ms}$ at all 14 time points, with the primary statistical analysis confirming no unacceptable prolongation of the QTcF interval (upper limits of the 90\% CIs $<10 \mathrm{~ms}$ at all time points; $p<0.001$ at all time points $[p$ values for the one-sided test of the $H_{0}$ of a mean difference $\geq 10$ ms against an $H_{\mathrm{A}}$ of a mean difference $\left.<10 \mathrm{~ms}\right]$ ) (Fig. 2). There was no significant exposure-response relationship between SNAC concentration and the QTcF interval; the estimated association between QTcF and SNAC concentration was 


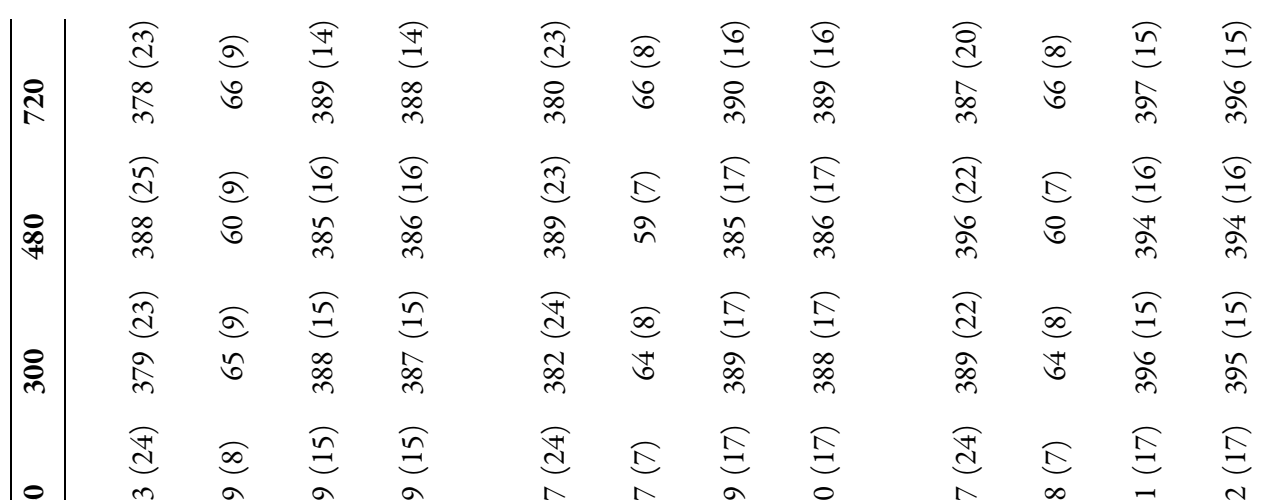

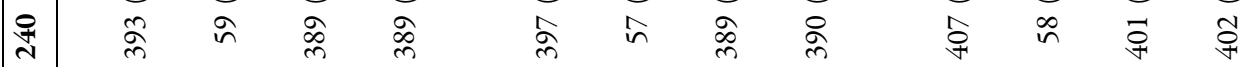

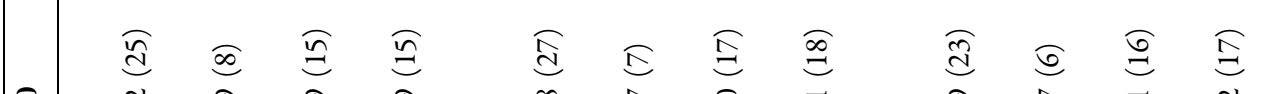

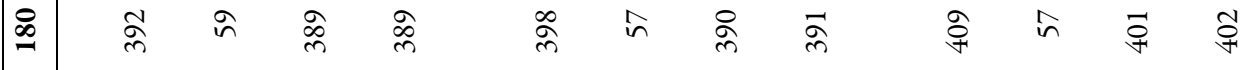

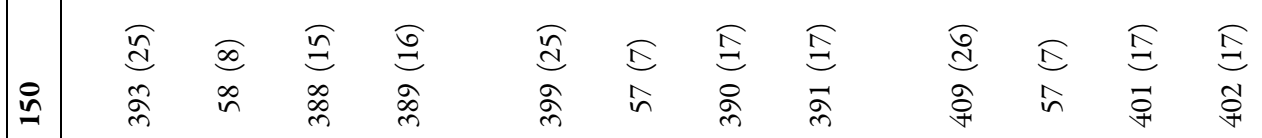

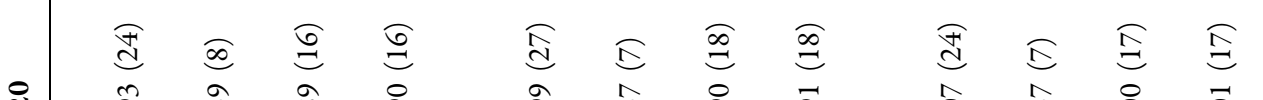

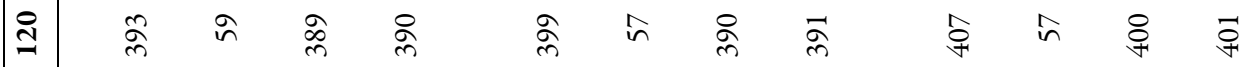

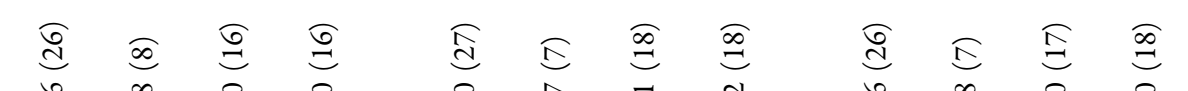

2 ¿

分方

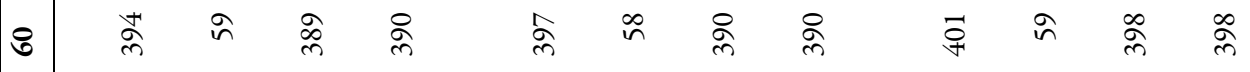

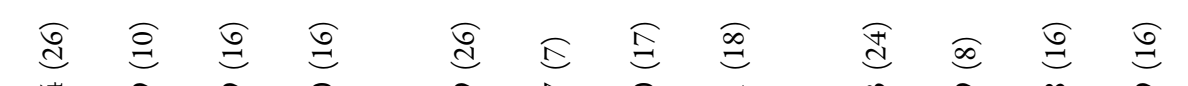

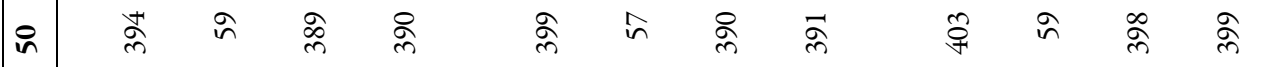

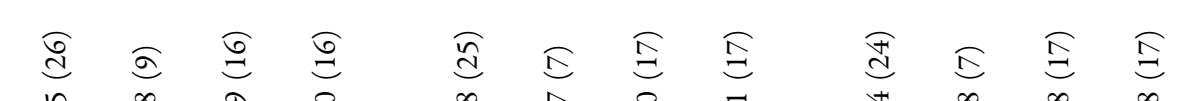

蓆

少

垈

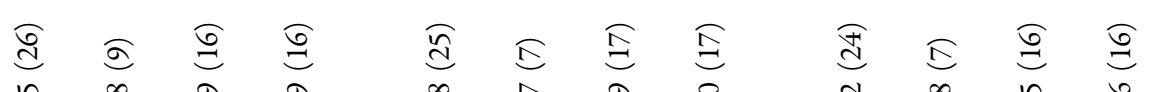

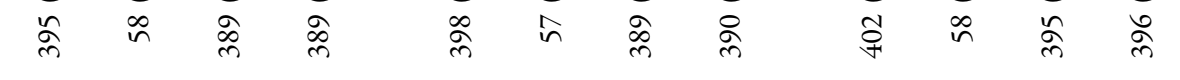

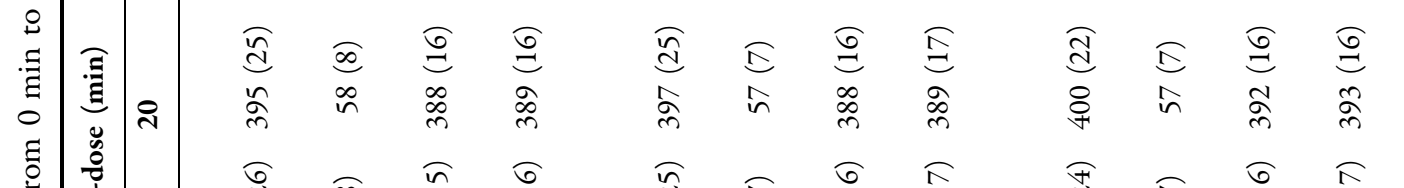

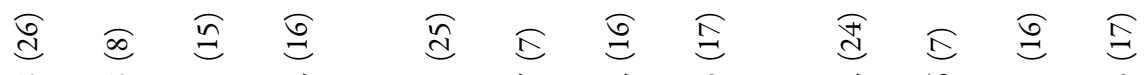

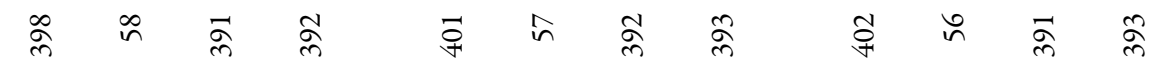

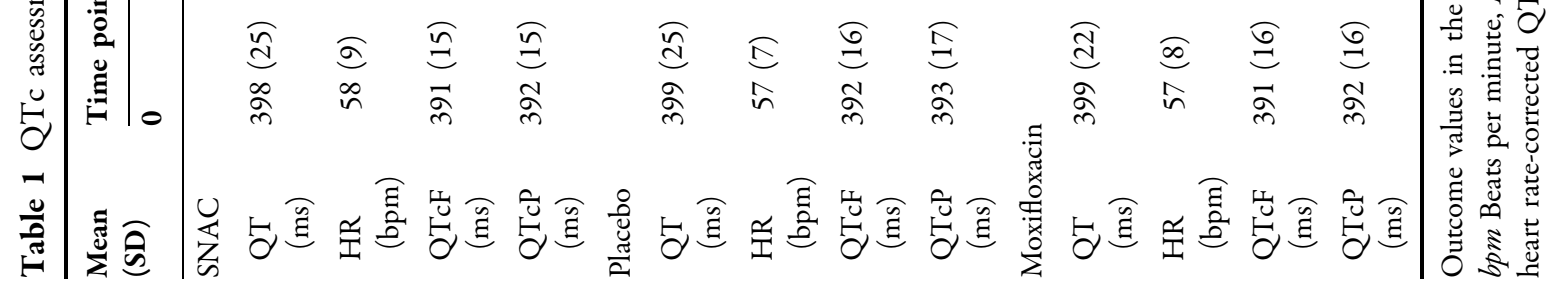

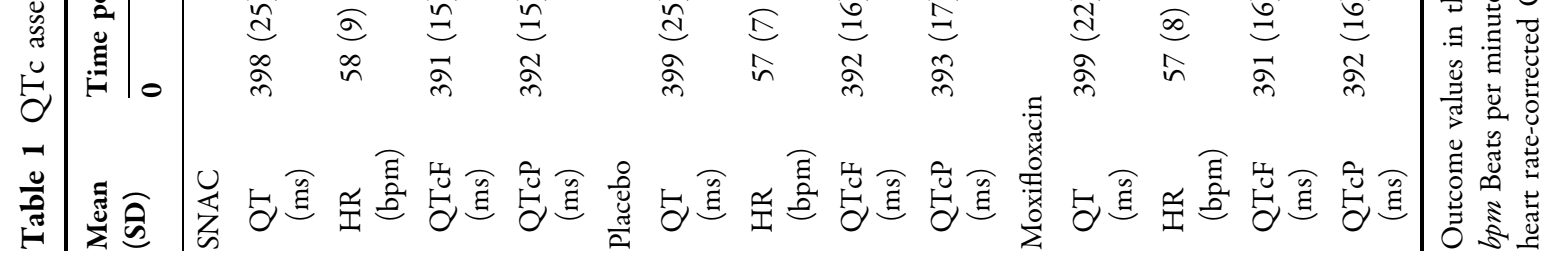




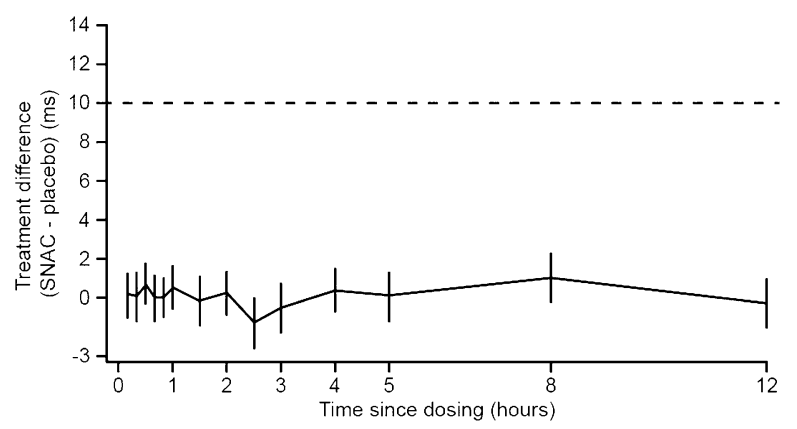

Fig. 2 Estimated mean time-matched baseline-adjusted difference between SNAC $3.6 \mathrm{~g}$ and placebo in the Fridericia heart rate-corrected QT interval (QTcF interval) following a single oral dose (full analysis set). Means are estimated from analysis of covariance models where the change from baseline for each of the $14 \mathrm{QTcF}$ endpoints were entered as a dependent variable, with subject, period and treatment as fixed effects and treatment period-specific baseline QTcF as covariate. Bars represent the corresponding two-sided $90 \%$ confidence intervals. The broken horizontal line is the threshold for the limit of interest. $S N A C$ Sodium N-(8-[2-hydroxy-benzoyl] amino) caprylate

$0.00007 \mathrm{~ms}$ per $\mathrm{ng} / \mathrm{mL}$ (95\% CI - 0.00006, 0.00020). The prespecified treatment difference (SNAC - placebo) in baseline-adjusted QTcF and $90 \% \mathrm{CI}$ at the mean $C_{\max }$ of SNAC was consistent with the primary analyses for both of the exposure-response analyses conducted. Hysteresis loop plots showed no sign of hysteresis. No clinically relevant changes in other ECG parameters (QTcP interval, QT interval, PR interval, QRS complex duration or HR) were observed with SNAC. The largest treatment difference in mean baseline-adjusted HR between SNAC and placebo at any time point was $2.1 \mathrm{bpm}(90 \%$ CI 1.1, 3.1).

The occurrence of outliers and abnormalities was low and similar between the SNAC and placebo treatment periods. None of the subjects experienced prolongation of QTc interval $>450 \mathrm{~ms}$ or increases from baseline $>30 \mathrm{~ms}$ or $\mathrm{HR}>100 \mathrm{bpm}$. Three subjects experienced PR intervals $>200 \mathrm{~ms}$ after treatment with either SNAC $3.6 \mathrm{~g}$ or placebo; all of these subjects had a pre-dose PR interval $>200 \mathrm{~ms}$. Of the three subjects who had QRS complex

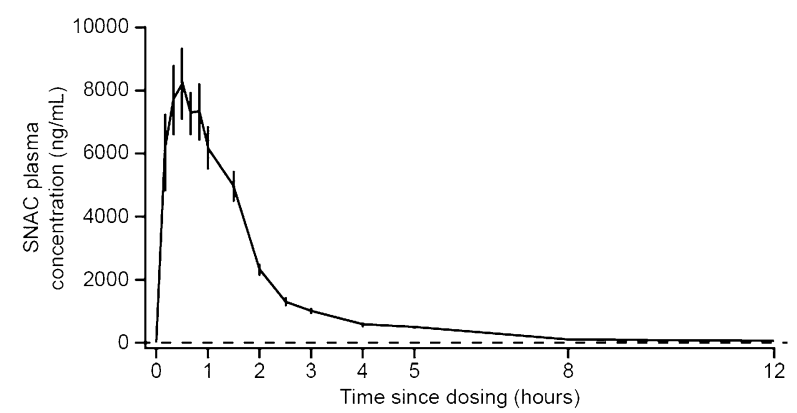

Fig. 3 Arithmetic mean plasma concentration-time profile following a single oral dose of SNAC $3.6 \mathrm{~g}$ (full analysis set). Bars represent the standard error of the mean. The broken horizontal line is the reference line for the lower limit of quantification. SNAC Sodium N-(8-[2-hydroxybenzoyl] amino) caprylate. $S N A C$ Sodium N-(8-[2-hydroxy-benzoyl] amino) caprylate

duration $>110 \mathrm{~ms}$ after treatment with either SNAC $3.6 \mathrm{~g}$ or placebo, two had QRS complex duration above this threshold pre-dose and the remaining subject had QRS complex duration $>110 \mathrm{~ms}$ at $12 \mathrm{~h}$ post-dose during all four treatment periods. T-wave abnormalities were reported in two subjects (in one subject during the SNAC period and one placebo period and in the other subject during one placebo period); there were no U-wave abnormalities reported.

\section{Part B-Pharmacokinetics}

The plasma concentration-time profile following a single dose of SNAC $3.6 \mathrm{~g}$ is shown in Fig. 3. Geometric mean (coefficient of variation [CV]) $\mathrm{AUC}_{0-12 \mathrm{~h}}$ and $C_{\max }$ values were 14,440 (37.0) $\mathrm{ng}^{*} \mathrm{~h} / \mathrm{mL}$ and 10,060 (75.0) $\mathrm{ng} / \mathrm{mL}$, respectively. Median $t_{\max }$ was 0.63 (minimum 0.18 , maximum 1.53) $\mathrm{h}$ and the geometric mean $t_{1 / 2}$ was $2.1(\mathrm{CV} 33.5) \mathrm{h}$. Five SNAC metabolites were investigated, all of which reached $C_{\max }$ within $5 \mathrm{~h}$, with geometric mean $t_{1 / 2}$ ranging from 2.6 to $4.0 \mathrm{~h}$.

\section{Part B-Safety and Tolerability}

Treatment-emergent AEs occurred in 12 subjects $(26.1 \%)$ during the SNAC treatment period (19 AEs), in seven subjects (14.6\%) during 
placebo treatment period 1 (8 AEs), in four subjects $(8.5 \%)$ during placebo treatment period 2 (7 AEs) and in four subjects (12.8\%) during the moxifloxacin treatment period (7 AEs). All AEs during the SNAC treatment period were mild or moderate in severity and no serious AEs were reported. One subject discontinued treatment in the second placebo treatment period due to an AE (anal abscess). One subject was withdrawn by the investigator on dosing day of treatment period 2 (after period 1 on placebo) due to QTcF prolongation in pre-dose ECGs which was judged to pose an unacceptable risk to the subject. The most frequently reported AEs in the SNAC treatment period were gastrointestinal and nervous system disorders, including nausea $(N=5)$, diarrhoea $(N=3)$, vomiting $(N=1)$, abdominal pain $(N=1)$, dizziness $(N=3)$ and headache $(N=3)$. No clinically relevant changes in safety laboratory parameters or other safety assessments were observed.

\section{DISCUSSION}

In the present study, a dose of SNAC that was 12-fold higher than that contained in oral semaglutide was not associated with an unacceptable prolongation of the QTcF interval, and assay sensitivity was confirmed using moxifloxacin as a positive control. Consistent with this result, there was no exposure-response relationship between SNAC concentration and QTcF interval, and no clinically relevant changes in other ECG parameters were identified. The testing of a SNAC dose of $3.6 \mathrm{~g}$ in the QTc assessments (12-fold higher than that used in oral semaglutide) provides reassurance of the absence of QTc prolongation at clinical dosages.

These findings complement those of a thorough QT/QTc study with s.c. semaglutide in 168 healthy subjects [15]. In the s.c. semaglutide study, there was no prolongation of the QTC interval at a supratherapeutic dose, with the upper limits of two-sided $90 \%$ CIs of the timematched baseline-adjusted differences between semaglutide and placebo in QTcF and QT interval corrected individually for HR (QTcI) observed to be $<10 \mathrm{~ms}$ at all time points. The results of this s.c. semaglutide study and the present SNAC study taken together suggest an absence of unacceptable prolongation of the QTc interval with oral semaglutide. Indeed, the cardiovascular safety of oral semaglutide has been demonstrated in a large outcomes trial in patients with type 2 diabetes and established cardiovascular disease or high cardiovascular risk [20].

No safety or tolerability concerns were identified after the administration of single oral doses of SNAC up to $3.6 \mathrm{~g}$. Throughout both parts of the study no serious or severe treatment-emergent AEs were reported.

In the present study, SNAC was given as a single dose, which regulatory authorities deem to be sufficient for products with short half-lives in QT/QTc studies [14]. Fridericia's correction was used to correct QT because QTcF is used for drugs that do not affect HR. The use of this QT correction formula is in line with the regulatory authority guidelines, suggesting that Fridericia's correction should be used to evaluate ECG data for all new drug applications [14].

Regulatory authorities suggest that supratherapeutic doses of drugs should be tested in QT/QTc studies. A limitation of this trial is that it is not possible to perform a single study with supratherapeutic doses of both the semaglutide and SNAC components, because oral semaglutide tablets contain a fixed dose of SNAC (300 mg) which would not give adequate exposure for QT/QTc assessment. Administering enough oral semaglutide tablets to achieve the dose of SNAC required for this study would mean that subjects would also receive a dose of the active pharmaceutical ingredient semaglutide far greater than is necessary and what would be acceptable from a gastrointestinal tolerability perspective based on a single dose administration. To overcome this, the results of the present trial (assessing the SNAC component) should be interpreted alongside the previously reported results from the supratherapeutic s.c. semaglutide study [15].

\section{CONCLUSIONS}

In conclusion, this was a thorough QT/QTc trial in which SNAC did not result in an 
unacceptable prolongation of the QTcF interval and there was no exposure-response relationship between SNAC concentration and QTcF interval. Assay sensitivity was demonstrated with moxifloxacin as the positive control.

\section{ACKNOWLEDGEMENTS}

The authors would like to thank all subjects who participated in this study, the study sites staff, and Marianne Bach Treppendahl (Novo Nordisk A/S) for critically reviewing the manuscript.

Funding. This study was funded by Novo Nordisk A/S. The study sponsor is also funding the journal's rapid service fee.

Authorship. All named authors meet the International Committee of Medical Journal Editors (ICMJE) criteria for authorship for this article, take responsibility for the integrity of the work as a whole, and have given their approval for this version to be published. All authors had full access to all of the data in this study and take complete responsibility for the integrity of the data and accuracy of the data analysis.

Authorship Contributions. Charlotte Granhall, Tine A. Bækdal, Flemming L. Søndergaard, Thomas W. Anderson and Mette Thomsen were involved in the concept and design of the study, Flemming L. Søndergaard performed the statistical analysis and Astrid Breitschaft collected the data. All authors contributed to the interpretation of the data and drafting of the manuscript.

Medical Writing, Editorial, and Other Assistance. Editorial support provided by Debbie Day and Andy Bond of Axis, a division of Spirit Medical Communications Group Ltd, was funded by Novo Nordisk A/S.

Disclosures. Charlotte Granhall, Tine A. Bækdal, Flemming L. Søndergaard, Thomas W. Anderson and Mette Thomsen are or were Novo Nordisk employees and own shares/stock. Astrid Breitschaft has nothing to disclose. Charlotte
Granhall and Thomas W. Anderson were employees of Novo Nordisk A/S at the time of the conduct of the trial and manuscript preparation.

Compliance with Ethics Guidelines. The trial was conducted in accordance with local regulatory requirements, good clinical practice guidelines and the Declaration of Helsinki. Approval from the Institutional Review Board/ Independent Ethics Committee (Landesamt für Gesundheit und Soziales Berlin, Ethik-Kommission des Landes Berlin, Berlin) was obtained prior to the commencement of the trial.

Data Availability. The datasets generated during and/or analysed during the current study are available from the corresponding author on reasonable request.

Open Access. This article is licensed under a Creative Commons Attribution-NonCommercial 4.0 International License, which permits any non-commercial use, sharing, adaptation, distribution and reproduction in any medium or format, as long as you give appropriate credit to the original author(s) and the source, provide a link to the Creative Commons licence, and indicate if changes were made. The images or other third party material in this article are included in the article's Creative Commons licence, unless indicated otherwise in a credit line to the material. If material is not included in the article's Creative Commons licence and your intended use is not permitted by statutory regulation or exceeds the permitted use, you will need to obtain permission directly from the copyright holder. To view a copy of this licence, visit http://creativecommons.org/licenses/by$\mathrm{nc} / 4.0 /$.

\section{REFERENCES}

1. Levin PA, Nguyen H, Wittbrodt ET, Kim SC. Glucagon-like peptide- 1 receptor agonists: a systematic review of comparative effectiveness research. Diabetes Metab Syndr Obes. 2017;10:123-39. 
2. European Medicines Agency. Byetta summary of product characteristics. March 2020. https://www. ema.europa.eu/en/documents/product-

information/byetta-epar-product-information_en. pdf. Accessed 25 Jan 2021.

3. European Medicines Agency. Eperzan summary of product characteristics. February 2019. https:// www.ema.europa.eu/en/documents/productinformation/eperzan-epar-product-information_en. pdf. Accessed 25 Jan 2021.

4. European Medicines Agency. Lyxumia summary of product characteristics. January 2021. https://www. ema.europa.eu/en/documents/productinformation/lyxumia-epar-product-information en.pdf. Accessed 25 Jan 2021.

5. European Medicines Agency. Ozempic summary of product characteristics. January 2021. https://www. ema.europa.eu/en/documents/productinformation/ozempic-epar-product-information en.pdf. Accessed 25 Jan 2021.

6. European Medicines Agency. Trulicity summary of product characteristics. November 2019. https:// www.ema.europa.eu/en/documents/productinformation/trulicity-epar-product-information_ en.pdf. Accessed 25 Jan 2021.

7. European Medicines Agency. Victoza summary of product characteristics. November 2019. https:// www.ema.europa.eu/en/documents/productinformation/victoza-epar-product-information_en. pdf. Accessed 25 Jan 2021.

8. European Medicines Agency. Rybelsus summary of product characteristics. December 2020. https:// www.ema.europa.eu/en/documents/productinformation/rybelsus-epar-product-information_ en.pdf, Accessed 25 Jan 2021.

9. Ma S, We L, Yang H, Deng S, Jevnikar AM. Emerging technologies to achieve oral delivery of GLP-1 and GLP-1 analogs for treatment of type 2 diabetes mellitus (T2DM). Can J Biotech. 2017;1(1):1-10.

10. Araújo F, Fonte P, Santos HA, Sarmento B. Oral delivery of glucagon-like peptide-1 and analogs: alternatives for diabetes control? J Diabetes Sci Technol. 2012;6(6):1486-97.

11. Buckley ST, Bækdal TA, Vegge A, et al. Transcellular stomach absorption of a derivatized glucagon-like peptide-1 receptor agonist. Sci Transl Med. 2018;10(467):e7047. https://doi.org/10.1126/ scitranslmed.aar7047.
12. Castelli MC, Friedman K, Sherry J, et al. Comparing the efficacy and tolerability of a new daily oral vitamin B12 formulation and intermittent intramuscular vitamin B12 in normalizing low cobalamin levels: a randomized, open-label, parallelgroup study. Clin Ther. 2011;33(3):358-71.e2.

13. Twarog C, Fattah S, Heade J, Maher S, Fattal E, Brayden DJ. Intestinal permeation enhancers for oral delivery of macromolecules: a comparison between salcaprozate sodium (SNAC) and sodium caprate (C(10)). Pharmaceutics. 2019;11(2):78.

14. International Conference on Harmonisation. ICH Harmonised Tripartite Guideline E14. The clinical evaluation of QT/QTc interval prolongation and proarrhythmic potential for non-antiarrhythmic drugs. May 2005. https://database.ich.org/sites/ default/files/E14_Guideline.pdf. Accessed 14 Jan 2021.

15. Demmel V, Sandberg-Schaal A, Jacobsen JB, Golor G, Pettersson J, Flint A. No QTc prolongation with semaglutide: a thorough QT study in healthy subjects. Diabetes Ther. 2018;9(4):1441-56.

16. International Conference on Harmonisation. ICH harmonised tripartite guideline. Good Clinical Practice. E6 (R1). Step 4. June 1996. https://www. ich.org/fileadmin/Public_Web_Site/ICH_Products/ Guidelines/Efficacy/E6/E6_R1_Guideline.pdf. Accessed14 Nov 2018.

17. World Medical Association. Declaration of Helsinki. Recommendations guiding physicians in biomedical research involving human subjects. Last amended by the 48th WMA General Assembly, Somerset West, Republic of South Africa, October 1996. https://www.wma.net/wp-content/uploads/2016/ 11/DoH-Oct1996.pdf. Accessed 8 July 2021.

18. Ring A, Walter B, Larbalestier L, Chanter D. An efficient crossover design for thorough QT studies. GMS Medizinische Informatik, Biometrie und Epidemiologie. 2010;6(1):1-12.

19. Ring A, Brand T, Macha S, et al. The sodium glucose cotransporter 2 inhibitor empagliflozin does not prolong QT interval in a thorough QT (TQT) study. Cardiovasc Diabetol. 2013;12:70.

20. Husain M, Birkenfeld AL, Donsmark M, et al. Oral semaglutide and cardiovascular outcomes in patients with type 2 diabetes. $\mathrm{N}$ Engl J Med. 2019;381(9):841-51. 